\title{
Utilidad del índice de resistencia obtenido por ultrasonido Doppler en nódulos tiroideos de características ecográficas malignas
}

\author{
Ángel Fernando Butrón-García y Mario Méndez-García \\ Instituto Mexicano del Seguro Social, Centro Médico Nacional del Noreste, Hospital de Especialidades 25, Departamento de Radiología e Imagen. \\ Monterrey, Nuevo León, México
}

\begin{abstract}
Resumen
Introducción: La patología nodular tiroidea tiene alta prevalencia en la población general. Su diagnóstico se ha incrementado con el Doppler, con el cual se pueden detectar características sugerentes de malignidad, como elevación de los índices de resistencia y pulsatilidad. Objetivo: Evaluar la correlación de un índice de resistencia alto y los hallazgos histopatológicos en pacientes con sospecha ecográfica de nódulos tiroideos malignos. Método: Estudio prospectivo, observacional, transversal y analítico de pruebas diagnósticas, en el Hospital de Especialidades 25, Monterrey, Nuevo León. Se incluyó a pacientes con nódulos tiroideos de características ecográficas malignas; se obtuvo el índice de resistencia de los nódulos, que se comparó con los hallazgos histopatológicos. Resultados: Se incluyeron 48 pacientes con diagnóstico ecográfico de nódulo tiroideo maligno. La correlación de índices de resistencia elevados y diagnóstico histopatológico de nódulos tiroideos malignos fue significativa $(p=0.001)$. La sensibilidad fue de $73.3 \%$, y la especificidad de $78.7 \%$; valor predictivo positivo de $61.1 \%$ y valor predictivo positivo negativo de $86.6 \%$. Conclusión: La determinación del índice de resistencia cada vez es más empleada, pero la sensibilidad y especificidad es baja y no es un método confiable, por lo que aún se requieren otros métodos complementarios.
\end{abstract}

PALABRAS CLAVE: Nódulo Tiroideo. Doppler. Cáncer de tiroides. Ecografía.

\begin{abstract}
Introduction: Thyroid nodular disease is highly prevalent in the general population. Its diagnosis has increased with Doppler ultrasound, by means of which features suggestive of malignancy can be detected, such as resistance and pulsatility indices elevation. Objective: To assess the correlation of a high resistance index and histopathological findings in patients with ultrasonographic suspicion of malignant thyroid nodules. Method: Prospective, cross-sectional, analytic, observational study of diagnostic tests carried out at Specialty Hospital 25, in Monterrey, Nuevo León. Patients with thyroid nodules of ultrasonographic malignant characteristics were included; the resistance index of the nodules was obtained and compared with histopathological findings. Results: Forty-eight patients with ultrasonographic diagnosis of malignant nodules were included. The correlation of elevated resistance indices with malignant thyroid nodule histopathologic diagnosis was significant $(p=0.001)$. Sensitivity was $73.3 \%$ and specificity $78.7 \%$; positive predictive value was $61.1 \%$ and negative predictive value was $86.6 \%$. Conclusion: The resistance index determination is increasingly being used, but its sensitivity and specificity are low, and it is not a reliable method, and other complementary methods are therefore still required.
\end{abstract}

KEY WORDS: Thyroid nodule. Doppler. Thyroid cancer. Ultrasonography.

Fecha de recepción: 10-02-2016

Fecha de aceptación: 26-04-2016

DOI://dx.doi.org/10.24875/GMM.18002417
Gac Med Mex. 2018;154:161-164

Disponible en PubMed www.gacetamedicademexico.com 


\section{Introducción}

La patología nodular tiroidea es una entidad de alta prevalencia en la población general, con mayor incidencia en mujeres. ${ }^{1}$ Su diagnóstico se ha incrementado exponencialmente desde la utilización del ultrasonido, ya que ha permitido detectar nódulos no palpables y ha aumentado la frecuencia de hallazgos incidentales en pacientes no seleccionados. ${ }^{2}$ La prevalencia estimada en la población general oscila entre 4 y $7 \%$, con una tasa más alta en las aéreas con deficiencia de yodo. Solo en Estados Unidos, cada año se detectan 275000 casos nuevos de nódulos tiroideos. ${ }^{3}$ El Estudio Framingham encontró que $4.2 \%$ de la población estudiada tenía nódulos en la evaluación inicial y que las mujeres tenían más nódulos en relación con los hombres (6.4\% de las mujeres versus $1.6 \%$ de los hombres); durante el seguimiento a los pacientes se encontró que $1.3 \%$ había desarrollado nodulos. ${ }^{4}$ El cáncer de tiroides es la neoplasia maligna más frecuente del sistema endocrino. La incidencia del cáncer de tiroides varía entre 1.2 y 10 entre 10000 pacientes, dependiendo del área geográfica, etnia y exposición previa a radiación, aumenta con la edad y alcanza una meseta después de los 50 años, aproximadamente. La detección de los nódulos no palpables se ha incrementado dramáticamente debido al ultrasonido, con el cual se ha revelado una prevalencia de nódulos tiroideos en la población general entre 19 y $46 \% .{ }^{5}$ Con este recurso diagnóstico, además de detectar nódulos con un diámetro menor a $1.5 \mathrm{~cm}$ se encontró que $62 \%$ de los clínicamente solitarios eran en realidad múltiples. ${ }^{6}$ Se calcula que su empleo ha cambiado la decisión terapéutica en $44 \%$ de los pacientes. En la actualidad, especialmente con el ultrasonido Doppler dúplex y Doppler poder se puede identificar características que sugieren malignidad: marcada hipoecogenicidad, vascularidad intranodular, halo periférico incompleto, márgenes irregulares, microcalcificaciones centrales, elevación de los índices de resistencia y pulsatilidad. En algunos estudios se han encontrado tasas de sensibilidad y especificidad de 77 y $79 \%$ para el Doppler, comparadas con la PAAF (punción-aspiración con aguja fina), la cual continúa siendo el método diagnóstico de elección. ${ }^{7}$

\section{Método}

Este estudio fue desarrollado con la información de pacientes de uno u otro sexo a quienes se les realizó ultrasonido y que fueron diagnosticados con nódulos tiroideos en el Hospital de Especialidades 25, Monterrey, Nuevo León. Los individuos con falta de interpretación del ultrasonido fueron excluidos del estudio y fueron eliminados de la investigación aquellos con reporte de muestra de patología insuficiente o cuyo reporte no estuvo en el periodo de investigación.

Los casos correspondieron a los pacientes que entre el 1 de julio y el 3 de octubre de 2014 cumplieron con los criterios descritos. A todos se les realizó ultrasonido Doppler y evaluación de los índices de resistencia. Los resultados fueron tabulados según la histología definitiva del nódulo y los hallazgos ecográficos. La significación estadística fue calculada por chi cuadrada.

El análisis descriptivo de las variables fue realizado con el Statistical Package for Social Sciences versión 17.0 (SPSS $®$ Inc., Chicago, IL, EE. UU.).

\section{Resultados}

Se encontraron 48 pacientes con diagnóstico ecográfico de nódulo tiroideo maligno, 42 pacientes (87.5 \%) fueron del sexo femenino. La edad media fue de 44.4 años, con una desviación estándar de 8.4 (Tabla 1).

El Doppler tiroideo mostró que 18 pacientes tuvieron un índice de alta resistencia, con una media de 0.98 y 30, así como un índice de baja resistencia con una media de 0.62. Mediante estudio histopatológico se confirmó que, de los 18 pacientes con índice de alta resistencia, 11 dieron positivo a algún tipo de neoplasia maligna de tiroides; de los 30 pacientes con

Tabla 1. Resultados de la prueba diagnóstica en la utilidad del índice de resistencia obtenido por ultrasonido Doppler en nódulos tiroideos de características ecográficas malignas

\begin{tabular}{lcc}
\hline & Valor absoluto & Porcentaje \\
\hline Sexo & 42 & 87.5 \\
Femenino & 6 & 12.5 \\
Masculino & & \\
Histología & 9 & 60.0 \\
$\quad$ Cáncer folicular & 5 & 33.3 \\
Cáncer papilar & 1 & 6.7 \\
Cáncer medular & & 73.3 \\
Sensibilidad & & 78.7 \\
Especificidad & & 61.1 \\
Valor predictivo positivo & & 86.6 \\
Valor predictivo negativo & & \\
\hline
\end{tabular}


índice de resistencia bajo, cuatro fueron positivos a malignidad: tres con cáncer papilar y uno medular. En cuando a la estirpe histopatológica de la neoplasia, nueve pacientes $(60 \%)$ tuvieron reporte de cáncer papilar, cinco (33.3) de cáncer folicular y uno (6.7\%) de medular (Tabla 1).

Se encontró diferencia significativa $(p=0.001)$ al comparar la proporción de pacientes con hallazgos de índice elevado de resistencia y diagnóstico histopatológico maligno de nódulos tiroideos. La sensibilidad del valor del índice de resistencia elevado para el diagnóstico de neoplasia maligna en los nódulos con evidencia ecográfica de malignidad fue de $73.3 \%$ y la especificidad fue de $78.7 \%$, con un valor predictivo positivo de $61.1 \%$ y un valor predictivo negativo de $86.6 \%$ (Tabla 1).

\section{Discusión}

En la mayoría de los pacientes con nódulos en la región anterior del cuello se debe considerar el origen tiroideo, si bien no hay que descartar otras posibilidades. El primer paso, una vez corroborado el origen tiroideo, es realizar evaluación clínica minuciosa que permita detectar factores de riesgo, síntomas y signos sugestivos de malignidad y disfunción tiroidea.

Entre los antecedentes personales, además de la historia de padecimientos tiroideos debe investigarse radiación a cabeza o cuello (factor que incrementa la incidencia de enfermedad tiroidea nodular no maligna) o exposición accidental a radiación antes de los 18 años. ${ }^{8}$ Es importante conocer el tiempo de evolución del nódulo, la velocidad del crecimiento nodular, la existencia de linfadenopatía cervical u otros síntomas, como dolor u obstrucción digestiva. En ausencia de bocios grandes, los datos de compresión traqueal, como tos y disfonía, sugieren lesión maligna. Igualmente es necesario investigar datos clínicos de disfunción tiroidea y antecedentes recientes de infección de vías respiratorias altas o embarazo, que orientan a la posibilidad de tiroiditis.

El dolor súbito en el nódulo o hacia la región occipital generalmente se debe a hemorragia en un nódulo y puede acompañarse de aumento súbito del volumen del este; no obstante, ante crecimiento rápido de un nódulo es importante considerar la posibilidad de carcinoma anaplásico o linfoma. ${ }^{6}$

Actualmente, la ecografía aplicada al estudio de la tiroides tiene amplia aceptación y sirve de apoyo para el estudio de la patología, tanto difusa como focal; sin embargo, a pesar de múltiples intentos en identificar signos ecográficos para llegar a un diagnóstico etiológico ${ }^{9}$ aún es imposible precisar el carácter benigno o maligno de un nódulo tiroideo sólo mediante la ecografía convencional. ${ }^{10}$ La modalidad más importante en el diagnóstico del nódulo tiroideo solitario sigue siendo la PAAF, por su alta seguridad diagnóstica."

Los valores de velocidad sistólica y diastólica en los carcinomas papilares fueron menores que en el resto; hubo cinco casos de cáncer papilar y solo uno no mostró esta característica. Estos resultados concuerdan con la catalogación de los carcinomas papilares como hipervasculares que han formulado otros autores. ${ }^{12}$ Hay que tener en cuenta el índice de resistencia, expresión de la resistencia o impedancia vascular, de lo cual se deduce que en la tiroides, a diferencia de lo observado en otros órganos,,$^{13}$ no es posible correlacionar los parámetros de resistencia vascular con el tipo de neoformación. ${ }^{8}$ El hecho de encontrar menor vascularización en los cánceres papilares podría explicar la escasa tendencia a la metástasis vía hemática de este tipo de tumores, así como su lento crecimiento, dada la relación directa entre angiogénesis y capacidad metastásica. ${ }^{14}$ Lo anterior se debe, según las últimas investigaciones sobre neovascularización, ${ }^{15}$ al hecho de que al presentar menos vasos existiría menor posibilidad de invasión del torrente sanguíneo. Este hecho podría atribuirse a la mínima producción de factores angiogénicos,$^{16}$ responsables de la formación de los vasos tumorales que a su vez permiten el crecimiento incontrolado de la neoformación, ${ }^{17}$ ya que se ha demostrado experimentalmente que un tumor sin neovascularización no crece más allá de $102 \mathrm{~mm} .{ }^{18}$

Los tipos histológicos concordaron con los registrados en otros estudios como el de Rivera Moscoso; el cáncer folicular fue el más frecuente. En cuanto a la sensibilidad del estudio fue muy baja, conforme a lo esperado por nosotros mismos, pero en comparación con la de otras investigaciones fue similar. ${ }^{18} \mathrm{La}$ especificidad de igual manera fue muy similar a la encontrada en la literatura. ${ }^{7}$ Al parecer, el método de imagen diagnóstica no es muy útil para diagnosticar, pero sí para orientar hacia dónde deben continuarse los estudios.

Quedaría para ulteriores investigaciones la comprobación de la existencia de una relación entre la escasa vascularización en los carcinomas papilares y una posible escasa cuantificación histológica de vasos neoformados, aunque existen autores que no han encontrado correlación entre las señales obtenidas mediante Doppler y la densidad vascular. ${ }^{5}$ Pensamos 
que estos resultados pueden constituir un paso más hacia la diferenciación entre malignidad y benignidad en el nódulo tiroideo, apoyada siempre en el resto de características diferenciales como vascularidad, contornos, microcalcificaciones hipoecoicas y contenido, teniendo presente la imposibilidad actual de sustituir por un método no invasivo la información obtenida mediante la PAAF. ${ }^{19}$

\section{Bibliografía}

1. Ezzat S, Sarti DA, Cain DR, Braunstein GD. Thyroid incidentalomas Prevalence by palpation and ultrasonography. Arch Intern Med. 1994;154(16):1838-1840.

2. Carroll BA. Asymptomatic thyroid nodules: incidental sonographic detection. AJR Am J Roentgenol. 1982:138(3):499-501.

3. Sriram U, Patacsil L. Thyroid Nodule. Dis Mon. 2004;50(9):486-526.

4. Kim N, Lavertu P. Evaluation of a thyroid nodule. Otolaryngol Clin North Am. 2003;36(1):17-33.

5. Gharib H, Papini E. Thyroid nodules: clinical importance, assessment, and treatment. Endocrynol Metab Clin North Am. 2007;36(3):707-735.

6. Rago T, Santini F, Scutari M, Pinchera A, Vitti P. Elastography: new developments in ultrasound for predicting malignancy in thyroid nodules. J Clin Endocrinol Metab. 2007;92(8):2917-2922.

7. De-Nicola H, Szejnfeld J, Logullo AF, Wolosker AM, Souza LR, Chiferi V. Flow pattern and vascular resistive index as predictors of malignancy risk in thyroid follicular neoplasms. J Ultrasound Med. 2005; 24(7):897-904.

8. Mazeh H, Beglaibter N, Prus D, Ariel I, Freund HR. Cytohistologic correlation of thyroid nodules. Am J Surg. 2007;194(2):161-163.
9. Berni A, Tromba L, Falvo L, Marchesi M, Grilli P, Peparini N. Malignant thyroid nodules: comparison between color Doppler diagnosis and histological examination of surgical samples. Chir Ital. 2002;54(5):643-647.

10. Kessler A, Gavriel H, Zahav S, Vaiman M, Shlamkovitch N, Segal S, et al. Accuracy and consistency of fine-needle aspiration biopsy in the diagnosis and management of solitary thyroid nodules. Isr Med Assoc J. 2005;7(6):371-373.

11. Peccin S, De-Castro JA, Furlanetto TW, Furtado AP, Brasil BA, Czepielewski MA. Ultrasonography: Is it useful in the diagnosis of cancer in thyroid nodules? J Endocrinol Invest. 2002;25(1):39-43.

12. Louvar E, Littrup PJ, Goldstein A, Yu L, Sakr W, Grignon D. Correlation of color Doppler flow in the prostate with tissue microvascularity. Cancer. 1998;83(1):135-140.

13. Sedelaar JP, De La Rosette J, Beerlage HP, Wijkstra H, Debruyne FM, Aarnink RG. Transrectal ultrasound imaging of the prostate: review and perspectives of recent developments. Prostate Cancer Prostatic Dis. 1999;2(5/6):241-252.

14. Frates $M C$, Benson $C B$, Charboneau JW, Cibas ES, Clark $\mathrm{OH}$, Coleman BG, et al. Management of thyroid nodules detected at US: Society of Radiologists in Ultrasound consensus conference statement. Radiology. 2005;237(3):794-800.

15. Frauscher F, Klauser A, Halpern EJ, Horninger W, Bartsch G. Detection of prostate cancer with microbubble ultrasound contrast agent. Lancet. 2001;357(9271):1849-1850.

16. Kelly IM, Lees WR, Rickards D. Prostate cancer and the role of color doppler US. Radiology. 1993;189(1):153-156.

17. Cochand-Priollet B, Guillausseau PJ, Chagnon S, Hoang C, Guillausseau-Scholer $C$, Chanson $P$, et al. The diagnostic value of fine-needle aspiration biopsy under ultrasonography in nonfunctional thyroid nodules: a prospective study comparing cytologic and histologic findings. Am J Med. 1994;97(2):152-157.

18. Solbiati L, Volterrani L, Rizzatto G, Bazzocchi M, Busilacci P, Candiani F et al. The thyroid gland with low uptake lesions: evaluation by ultrasound Radiology. 1985;155(1):187-91.

19. Jameson J. Trastornos de la glándula tiroides. En: Braunwald E, Hauser S, Fauci A, Longo D, Kasper D, Jameson JL, editores. Harrison. Principios de Medicina Interna. Volumen 2. España: McGraw-Hill; 2006. 